\title{
The Influence of Preparation Factors on Physical Characteristics of Chitosan Nanoparticles
}

\author{
Norra Shamiela Ruslan, ${ }^{1}$ Noratiqah Mohtar, ${ }^{1}$ Siti Sarah Fazalul Rahiman ${ }^{2}$ \\ and Amirah Mohd Gazzali ${ }^{*}$
}
${ }^{1}$ Department of Pharmaceutical Technology, School of Pharmaceutical Sciences, Universiti Sains Malaysia, 11800 USM Pulau Pinang, Malaysia ${ }^{2}$ Department of Physiology, School of Pharmaceutical Sciences, Universiti Sains Malaysia, 11800 USM Pulau Pinang, Malaysia

*Corresponding author: amirahmg@usm.my

Published online: 25 November 2020

To cite this article: Ruslan, N. S. et al. (2020). The influence of preparation factors on physical characteristics of chitosan nanoparticles. J. Phys. Sci., 31(3), 47-60. https://doi.org/10.21315/jps2020.31.3.4

To link to this article: https://doi.org/10.21315/jps2020.31.3.4

\begin{abstract}
Chitosan has been shown to have great potentials in various pharmaceuticals and biomedical applications, including drug delivery. Derived from chitin abundantly available in the shells of crustaceans such as crabs and shrimps, this naturally occurring polysaccharide is classified based on its molecular weight: low, medium or high. This study aimed to explore the production of chitosan nanoparticles (NP) and the influence of different factors on the physical properties of the NP produced. These factors were the concentrations of acetic acid, chitosan flakes and tripolyphosphate (TPP). The design of experiment (DoE) approach was used to determine the optimum conditions for the production of chitosan NP, with particle size ( $\mathrm{nm}$ ) and polydispersity index (PdI) being set as the responses. The chitosan flakes were solubilised in acetic acid at a specific concentration determined by the DoE before dropwise addition of TPP in an ice bath. The mixture was stirred at room temperature and subsequently centrifuged to remove the unformed materials, and then was spray-dried into powder. The size, surface charge, shape and morphology of the particles produced were characterised and infrared analysis was conducted. The results showed that the particles were spherical, slightly positively charged ( $\zeta$-potential: +2.89 at $\mathrm{pH} 7)$ and the infrared analysis displayed important peaks of the chitosan NP. The DoE results showed that not all combinations of parameters could produce NP; hence, determination of concentration for each parameter is essential. The equation produced by the DoE will be a useful guide to minimise error in this circumstance. In conclusion, the acetic acid and chitosan flakes concentrations were found to influence the particle size positively, whilst the increment in TPP concentration will adversely affect the particle size. Similar pattern of response was also observed for the PdI of the particles.
\end{abstract}


The methods used in this study has successfully produced spherical particles, with evidence of interactions between TPP and chitosan in the NP as shown in the infrared spectrum.

Keywords: Chitosan, nanoparticles, spray-dry, ionic-gelation, design of experiments

\section{INTRODUCTION}

Nanotechnology has gained a great deal of public interest due to the needs and applications of nanomaterials in many areas of human endeavours such as industry, agriculture, business, medicine and public health. Nanotechnology includes the integration of the nanoscale structures into larger material components and systems, whilst keeping the benefits of new and improved materials at a nanoscale level.

Nanotechnology provides a platform to formulate drugs in nanometer size range that would enhance their efficacy in a variety of dosage forms. Recent advancement in nanotechnology has proven that nanoparticles (NP) has great potentials as drug carriers. The interfacial layers surrounding the particles play an integral part in nanoscale matter, fundamentally affecting all their properties. These interfacial layers typically consist of ions, inorganic and organic molecules. ${ }^{1}$

Particle reduction technologies have enabled the production of different types of nanostructures that exhibit unique physicochemical and biological properties. These particles are being widely explored for various biomedical applications and acquiring significant importance the pharmaceutical field. Other advantages of small particles include reducing drug-related toxicity, enhancing drug release, improving solubility and bioavailability, and providing better formulation opportunities for the drugs. ${ }^{2}$

Targeted drug delivery is a useful delivery approach to improve the safety and efficacy of therapeutic agents. The application of NP has allowed the possibility of targeted drug delivery through enhanced permeability and retention (EPR) effect. Many organic and inorganic materials have been used to prepare NP as reported in the literature, with different characteristics and properties depending on the forming materials. ${ }^{3,4}$ Organic materials such as chitosan, poly(lactic-co-glycolic acid) (PLGA) and poly(hydroxyalkanoates) (PHA) are among the polymers that are being extensively explored for numerous applications alongside other inorganic materials such as zinc oxide and graphene.,

Chitosan is a hydrophilic cationic linear polyamine derived from chitin, a substance that is found in abundance in crustacean shells such as crabs, shrimps and lobsters. It is a biodegradable polysaccharide, containing both glucosamine 
and $N$-acetyl- $D$-glucosamine (NAG) as its main forming units. ${ }^{7}$ Unlike chitin that is insoluble in most solvents, chitosan is soluble in acidic environment. At $\mathrm{pH}$ lower than 6.5, the glucosamine units will convert into a soluble form. At higher $\mathrm{pH}$, however, chitosan will precipitate. The biodegradability, biocompatibility and low toxicity properties of chitosan have made it suitable to be used in formulating pharmaceuticals and biomedical preparations.

A substantial body of literature has reported the attractiveness of chitosan as $\mathrm{NP}$, which has prompted more research on its productions and applications. ${ }^{8}$ However, due to the different grades and molecular weight of chitosan available in the market, one fixed method would not be suitable for the production of NP from all types of chitosan. Since acid is needed to dissolve the chitosan flakes, the choice of acid at appropriate concentrations should also be considered in formulating the chitosan NP. Furthermore, the choice of crosslinking agent will also determine the characteristics of the NPs. Different crosslinking agents have been explored and reported in the literature and these include potassium persulfate (KPS), tripolyphosphate (TPP) and hexametaphosphate (HMP). ${ }^{9}{ }^{10}$ Taken together, the above-mentioned factors directly influence the physical properties of the NP and therefore, it is essentially important to optimise these factors to produce a functional chitosan NP.

This article describes the production of chitosan NP and the influence of different parameters on the physical properties of the NP produced. In this study, a range of acetic acid concentrations was used to solubilise the chitosan and TPP was employed as the crosslinking agent. Three factors were selected for evaluation, namely the concentrations of acetic acid, chitosan flakes and TPP. Design of experiments (DoE) approach was used to determine the optimum conditions required for the production of chitosan NP by using Design-Expert ${ }^{\circledR}$ statistical software. There was no specific target application being set at the beginning of the study to allow a wider possibility of chitosan NP applications. The specific future applications, however, could be tailored and enhanced accordingly based on the characteristics of the NP obtained from this study.

\section{EXPERIMENTAL}

\subsection{Materials}

Chitosan (low molecular weight or LMW, approximately $150000 \mathrm{Da}$ ) was obtained from Sigma-Aldrich (Malaysia). Acetic acid glacial was obtained from Systerm (Malaysia) whereas TPP sodium were purchased from R\&M (Malaysia). All chemicals were used as received. 


\subsection{Methodologies}

\subsubsection{Preparation of chitosan NP}

Unless specified, the chitosan NP were prepared as in the following discussion. Chitosan flakes were solubilised in acetic acid through stirring at room temperature. The chitosan solution was then placed in an ice bath and TPP was added dropwise under constant stirring. The unformed materials were separated through low speed centrifugation (4000 rpm, $10 \mathrm{~min}$ ) and the size of the NP present in the supernatant was determined before they were dried.

The drying process for the NP was conducted through the spray-drying technique. The supernatant containing chitosan NPs was spray-dried using the Mini Spray Dryer B-290 (Buchi Labortechnik AG, Switzerland) with a long-drying chamber, a spray cap and particle collector. The conditions were set at inlet air temperature of $80^{\circ} \mathrm{C}$, flow rate of $95-1051 \mathrm{~min}^{-1}$ and the relative spray rate was kept constant. The spray-dried nanoparticle powder was collected from the particle collector chamber using a scraper and was stored in the refrigerator $\left(4^{\circ} \mathrm{C}\right)$ until further use.

\subsubsection{Mixture design}

Design-Expert ${ }^{\circledR}$ software version 12 (Stat-Ease Inc., United States) was used to generate 11 experiments using mixture design (Table 1). The composition of three materials were evaluated, which were the percentage of chitosan (component A; $0.5 \%-3 \%$ ), acetic acid (component $\mathrm{B} ; 1 \%-5 \%$ ) and TPP (component $\mathrm{C}$; $0.5 \%-3 \%)$. The selected evaluated responses were particle size $(\mathrm{nm})$ and polydispersity index (PdI). The experiments were done in triplicate and the results were analysed using this software.

\subsubsection{Particle size characterisation using dynamic light scattering (DLS) technique}

The average particle size and PdI of NP was determined using the Zetasizer NanoZS (Malvern Instruments, United Kingdom) which uses the concept of DLS in the measurement. The analysis was conducted at a scattering light angle of $173^{\circ}$ and a temperature of $25^{\circ} \mathrm{C}$. The instrument was calibrated using polystyrene latex standard prior to the measurement. Unless specified, each sample was analysed by diluting the sample of NP suspension with deionised water at 1:10 ratio. Values reported are mean particles size \pm standard deviation. The PdI range of the particles was between 0 and 1 , and was recorded concurrently during measurement of the average particle size. 
Table 1: The particle size and PdI of chitosan NP produced from LMW chitosan.

\begin{tabular}{|c|c|c|c|c|c|}
\hline \multirow{3}{*}{ Run } & \multicolumn{3}{|c|}{ Component } & \multirow{2}{*}{\multicolumn{2}{|c|}{ Response }} \\
\hline & A & B & $\mathrm{C}$ & & \\
\hline & Acetic acid (\%) & Chitosan (\%) & ТPР (\%) & Particle size (nm) & PdI \\
\hline 1 & 2.5 & 3 & 0.5 & Particles did not form & - \\
\hline 2 & 5 & 0.5 & 0.5 & $329 \mathrm{~nm} \pm 5.8$ & $0.298 \pm 0.02$ \\
\hline 3 & 3.75 & 0.5 & 1.75 & $1393 \mathrm{~nm} \pm 476$ & $1.000 \pm 0$ \\
\hline 4 & 3.75 & 1.72 & 0.5 & Particles did not form & - \\
\hline 5 & 2.4 & 1.8 & 1.8 & Particles did not form & - \\
\hline 6 & 1 & 2 & 3 & Particles did not form & - \\
\hline 7 & 2.4 & 1.8 & 1.8 & Particles did not form & - \\
\hline 8 & 2 & 2 & 1.98 & Particles did not form & - \\
\hline 9 & 2.5 & 3 & 0.5 & Particles did not form & - \\
\hline 10 & 2.4 & 1.8 & 1.8 & Particles did not form & - \\
\hline 11 & 2.5 & 0.5 & 3 & $737 \mathrm{~nm} \pm 68$ & $0.595 \pm 0.07$ \\
\hline
\end{tabular}

Note: All experiments were conducted in triplicate $(n=3)$

\subsubsection{Determination of surface charge}

Zeta $(\zeta)$ potential was measured by using Zetasizer Nano-ZS (Malvern Instruments, United Kingdom). Each sample was measured three times at $25^{\circ} \mathrm{C}$ using a folded capillary cell with electrodes.

\subsubsection{FTIR spectroscopy}

The infrared spectrum of chitosan NP and the raw materials were obtained using the PerkinElmer Frontier ${ }^{\mathrm{TM}}$ MIR spectrometer. The recording was set at $4 \mathrm{~cm}^{-1}$ resolution, 32 scans with a transmission mode between $400 \mathrm{~cm}^{-1}$ and $6000 \mathrm{~cm}^{-1}$. The data obtained was analysed using Spectrum ${ }^{\circledR}$ software.

\subsubsection{Transmission electron microscopy (TEM)}

The morphology of the NP was analysed by the TEM (CM12 ${ }^{\circledR}$ FEI, Eindhoven, Netherlands). Diluted samples were dropped on a sample grid and was air-dried. It was then visualised under the microscope. 


\subsubsection{Scanning electron microscopy (SEM)}

The surface morphology of the dried NP was visualised using the ZEISS SUPRA ${ }^{\circledR}$ 50 VP SEM. The samples were pre-coated with a layer of gold $(\mathrm{Au})$ as a standard to improve image resolution and contrast in the field transmission system to produce a homogenous surface for imaging.

\section{RESULTS AND DISCUSSION}

Chitosan NP have been described to exhibit excellent physicochemical, antimicrobial and biological properties, making them a superior environmentally friendly nanomaterial besides possessing bioactivity that does not harm humans. ${ }^{8}$ Among the famous methods to produce chitosan NP include ionic gelation and ion-complexation methods in the presence of acids as the solvent. Chitosan is practically insoluble at a neutral or higher $\mathrm{pH}$ due to its $\mathrm{pKa}$, which ranges from 6.2 to 7.0 , implying that its protonation could only occur at a lower $\mathrm{pH}$. At such $\mathrm{pH}$, the $\mathrm{NH}_{2}$ groups that are abundantly present in the chitosan polymeric chains will be protonated, leading to the expansion of the chain due to the electrostatic interaction between the charged groups. ${ }^{11}$

In this study, a mixture design was used to find the optimal formulation of chitosan NP from 11 different combinations of the three components (as seen in Table 1). It is interesting to note that only 3 out of 11 formulas successfully produced particles, and all of these three formulas used $0.5 \% \mathrm{w} / \mathrm{v}$ of chitosan with variable quantity of TPP. It is also evident that at a constant concentration of chitosan, high quantity of TPP was not needed to produce monodisperse NP, as shown in Run \#2 in comparison to Run \#3 and Run \#11 (Table 1).

The results obtained were analysed using the Design-Expert ${ }^{\circledR}$ software and significant factors were included in the following coded equations:

$$
\begin{aligned}
\text { Particle size }= & 329.34 \mathrm{~A}+478.25 \mathrm{~B}-2330.81 \mathrm{C}-1798.01 \mathrm{AB} \\
& +8825.22 \mathrm{AC}+5467.00 \mathrm{BC}-23868.52 \mathrm{ABC} \\
\mathrm{PdI}= & 0.2983 \mathrm{~A}+0.4297 \mathrm{~B}-1.36 \mathrm{C}-1.62 \mathrm{AB}+5.68 \mathrm{AC} \\
+ & 2.95 \mathrm{BC}-15.41 \mathrm{ABC}
\end{aligned}
$$

A positive value in the equations will lead to an increase in the response, and vice versa. As an example, an increase in the concentration of chitosan (positive value, Component A) will cause a direct increment in the particle size, whilst an 
increase in the concentration of TPP (negative value, Component C) will produce a smaller particle size (Equation 1). The 3D surface graphs (Figure 1) also showed the influence of these factors towards both responses (i.e., particle size and PdI).

(A)
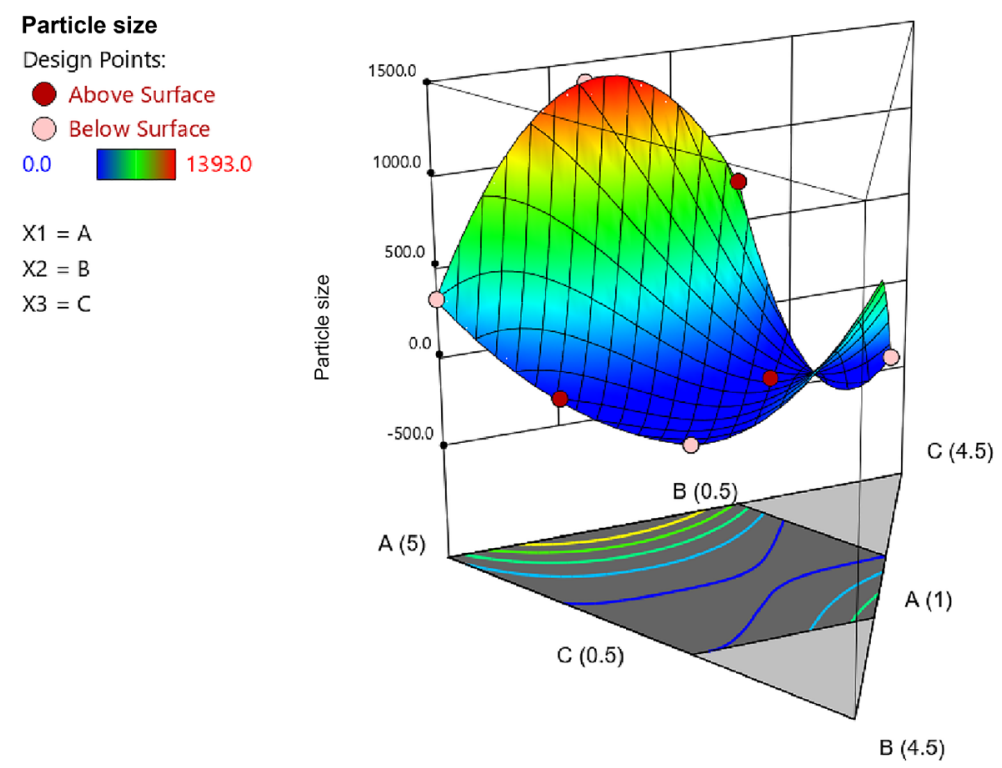

(B) Pdl

Design Points:

Above Surface

Below Surface

$0.000 \square 1.000$

$\mathrm{X} 1=\mathrm{A}$

$\mathrm{X} 2=\mathrm{B}$

$\mathrm{X} 3=\mathrm{C}$

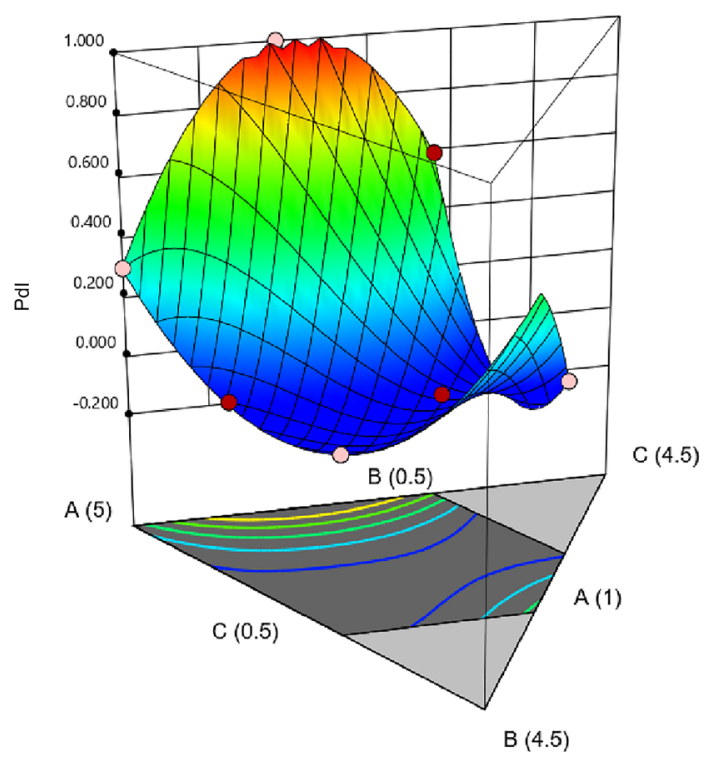

Figure 1: 3D surface graphs for (A) particle size and (B) PdI. 
The choice of crosslinking agent is an important factor that influences the size and properties of the chitosan NP produced. TPP is a well-known non-toxic agent and is suitable for drug delivery purposes. During the production process, TPP, which is a polyanionic crosslinking agent, would interact with the positively charged $\mathrm{NH}_{2}$ groups and form complexes, leading to the formation of the NP. ${ }^{12}$ This process will eventually neutralise the chitosan surface charge, producing neutral or slightly charged particles. This was demonstrated in this study as the surface charge of the prepared chitosan NP was slightly positive ( $\zeta$-potential: +2.89 at $\mathrm{pH} 7$ ). Previously, it has been reported that particles with slight charges tend to accumulate more efficiently in tumour cells, hence, the chitosan NP produced in this study have great potentials to be further explored as a delivery agent towards tumour cells. ${ }^{9}$

FTIR analysis was conducted on the chitosan flakes, the TPP and the chitosan NP. The spectrum obtained are presented in Figure 2 and the main peaks corresponding to the important functional groups present in the tested materials are summarised in Table 2.

The structure of chitosan is presented in Figure 3. The abundance of -OH and -NH groups have contributed to the broad peak at $3423.16 \mathrm{~cm}^{-1}$, which corresponded to the stretching vibration of O-H and N-H. TPP, on the other hand, has oxygen and phosphorus, which translated into peaks that are specific to the vibrations between the two atoms (Table 2).

Table 2: The main FTIR peaks recorded for chitosan flakes, TPP and chitosan NP.

\begin{tabular}{lll}
\hline Material & Wavenumber $\left(\mathrm{cm}^{-1}\right)$ & Corresponding functional group \\
\hline Chitosan & 3423.16 broad & N-H/O-H stretching vibration \\
& 2922.34 & $\mathrm{C}-\mathrm{H}$ stretching \\
1654.33 & $\mathrm{C}=\mathrm{O}$ \\
1421.94 & $\mathrm{O}-\mathrm{H}$ deformation bending vibration \\
1382.88 & $\mathrm{C}-\mathrm{H}$ bending vibration \\
1076.99 & $\mathrm{C}-\mathrm{O}$ stretching vibration \\
& 1212.94 & $\mathrm{P}=\mathrm{O}$ stretching vibration \\
TPP & 1144.27 & $\mathrm{O}-\mathrm{P}=\mathrm{O}$ stretching vibration \\
& 1095.49 & $\mathrm{PO}$ stretching vibration \\
& 896.17 & $\mathrm{P}-\mathrm{O}-\mathrm{P}$ stretching vibration \\
Chitosan NP & 3440.93 broad & N-H/O-H stretching vibration \\
& 3288.14 & $\mathrm{P}-\mathrm{NH}$ stretching \\
& 1640.75 & $\mathrm{~N}-\mathrm{H}$ bending vibration \\
& 1561.77 & $\mathrm{~N}-\mathrm{O}-\mathrm{P}$ stretching vibration \\
\hline
\end{tabular}



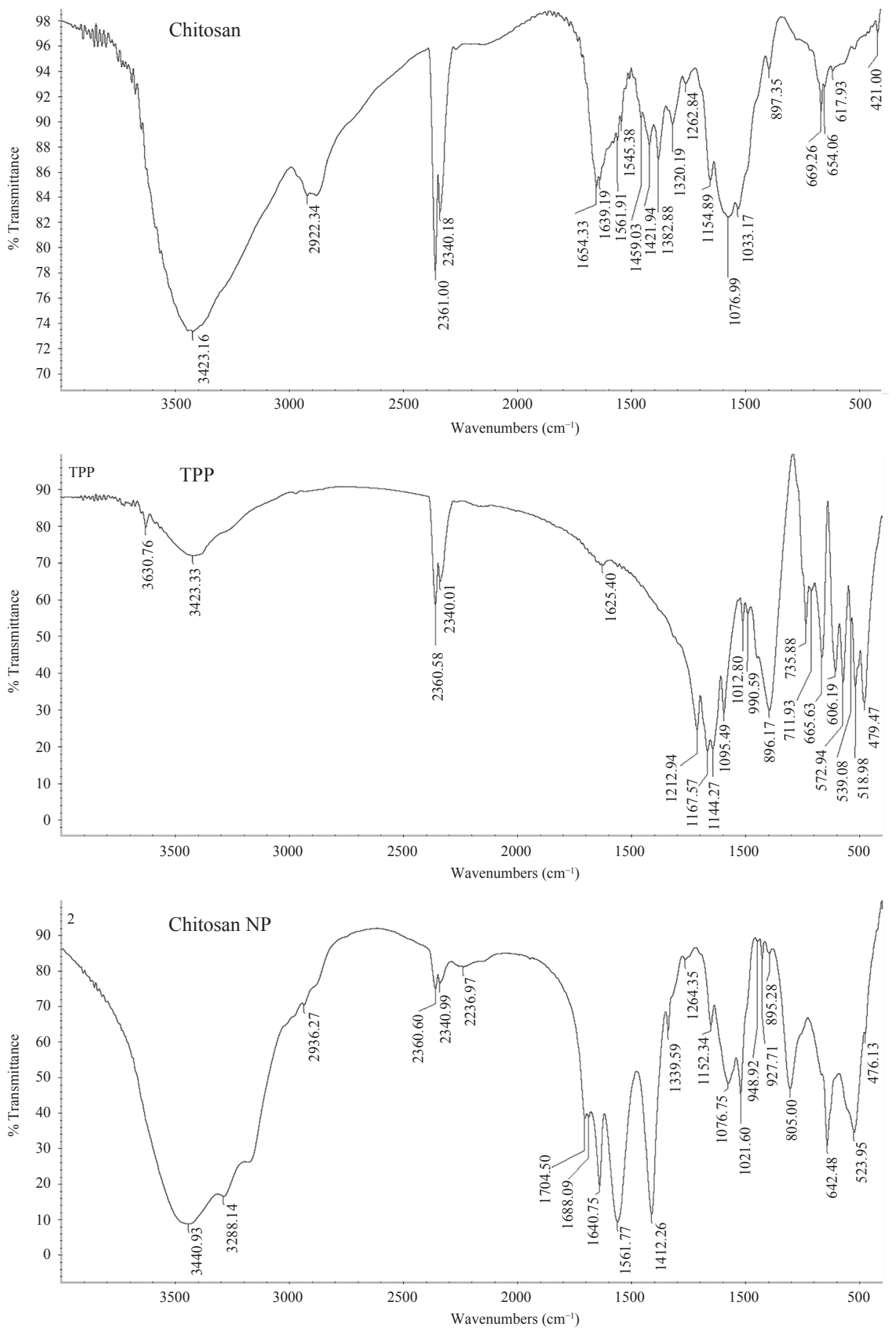

Figure 2: The FTIR spectrum of chitosan flakes, TPP and chitosan NP. 


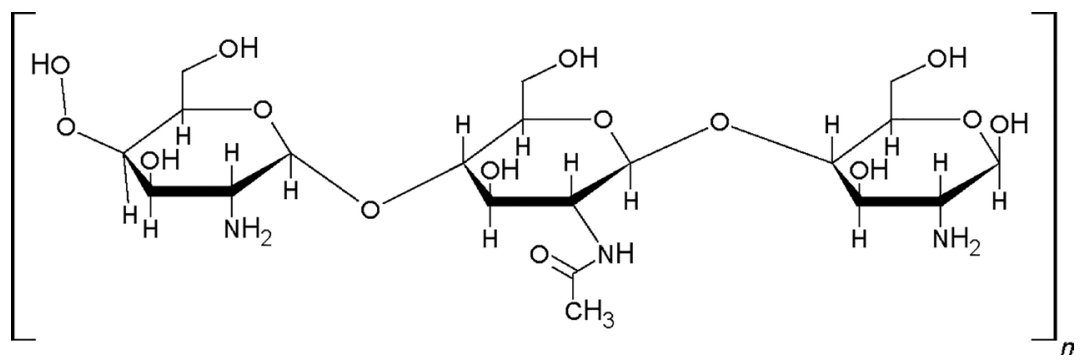

Figure 3: Molecular structure of chitosan.

The chitosan NP spectra showed the shift of the $\mathrm{O}-\mathrm{H} / \mathrm{N}-\mathrm{H}$ vibration to $3440.93 \mathrm{~cm}^{-1}$. In addition, a new peak emerged at $3288.14 \mathrm{~cm}^{-1}$, corresponding to the stretching vibration of P-NH. The appearance of a new peak at $1561.77 \mathrm{~cm}^{-1}$ that was assigned to the stretching vibration of N-O-P, further confirmed the interaction between chitosan and TPP in the chitosan NP produced. As displayed in Figure 4, the interaction between TPP and the protonated $\mathrm{NH}_{2}$ groups led to the formation of chitosan NP, as previously described in the literature. ${ }^{13}$

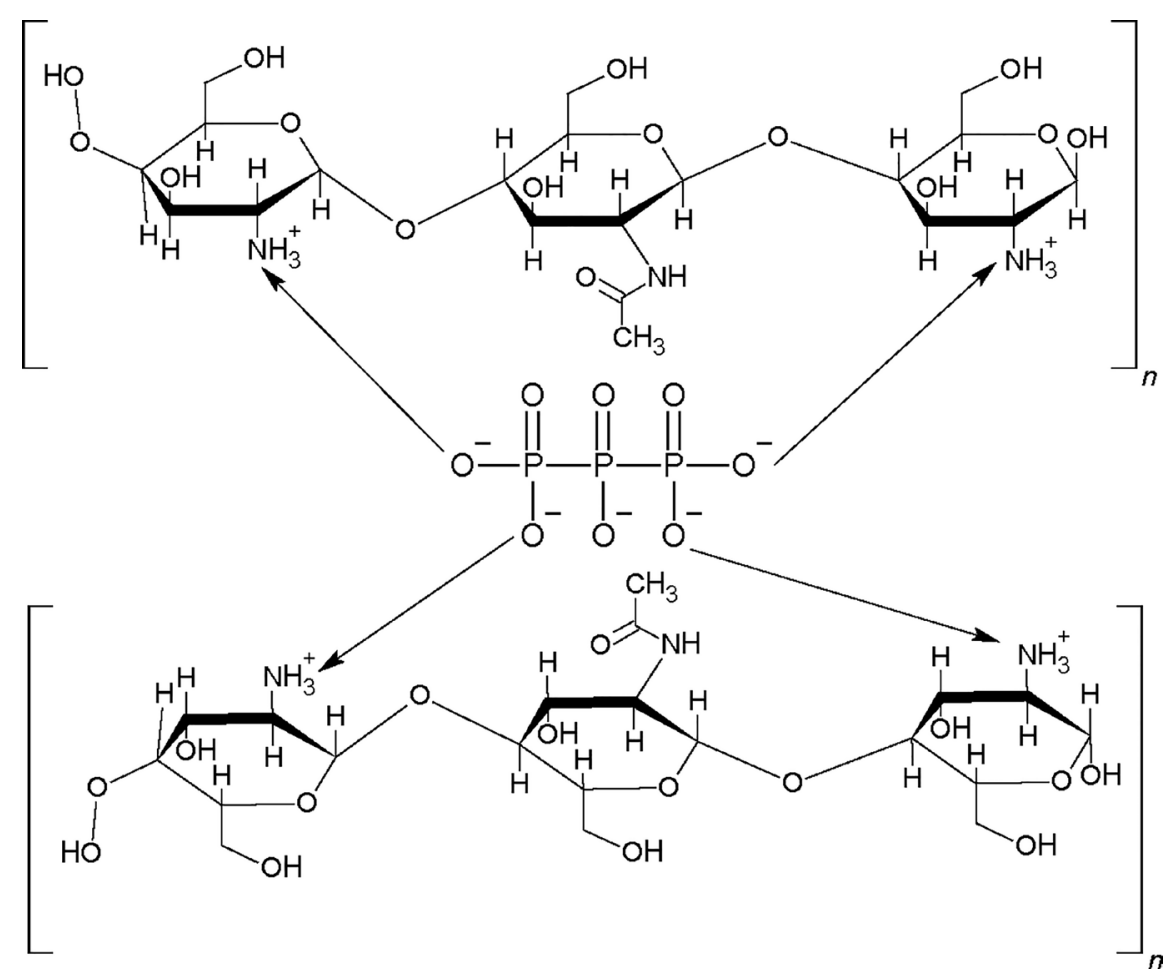

Figure 4: The crosslinking interaction between chitosan and TPP. ${ }^{13}$ 
In the production of NP, the shape of the particles need to be determined as this factor would influence the fate of the particles in the body and their interactions with cells. ${ }^{14,15}$ Based on the TEM analysis, the shape of the chitosan NP produced are spherical (Figure 5). This is in accordance with the theory on the formation of chitosan NP as reported by Mohammed et al. ${ }^{16}$ The authors described that the formation of chitosan NP takes place following the interaction and complexation between positively charged chitosan and negatively charged polyanionic crosslinker during mechanical stirring. This interaction would lead to the separation of the NP in spherical shape with different sizes and specific surface charges, depending on the process parameters. ${ }^{16}$
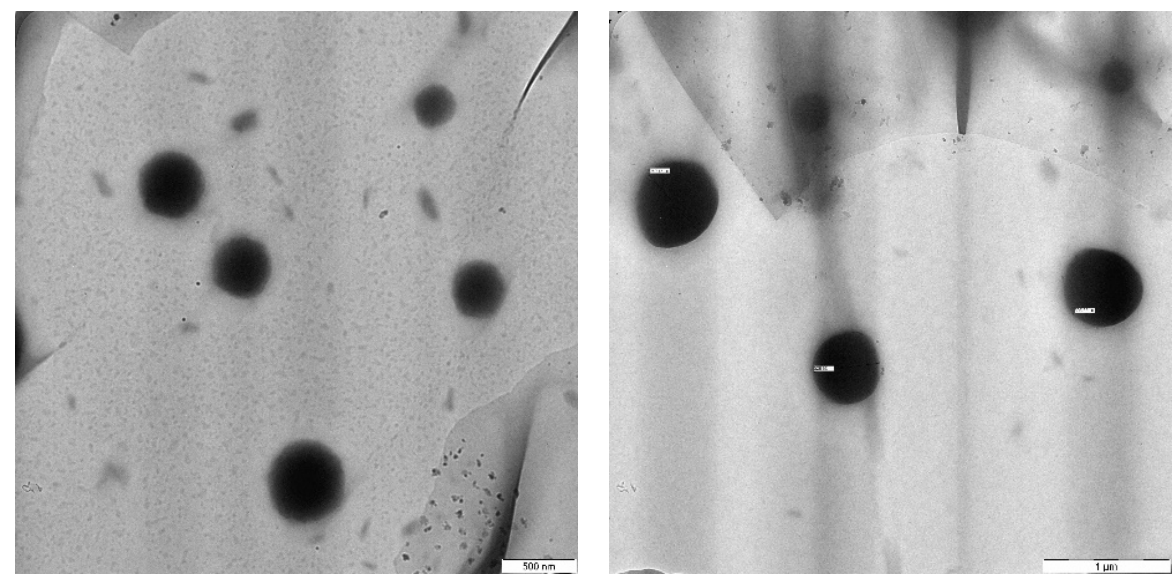

Figure 5: TEM images of chitosan NP produced.

The spray-drying technique utilised in this study may not be suitable for LMW chitosan since the particles produced were found to be crumpled based on the SEM images obtained (Figure 6). The presence of heat during the process may be unfavourable to these particles. However, a preliminary study on the production of chitosan NP from high molecular weight (HMW) chitosan using spray-drying technique showed that intact spherical particles were produced at the end of the drying process (data not shown). This finding implies that the type of chitosan used in the production of NP may behave differently during the drying process. An improvement in the drying technique would be necessary to preserve the shape of the NP from LMW chitosan through other methods such as sugar coating prior to spray-drying or lyophilisation. 


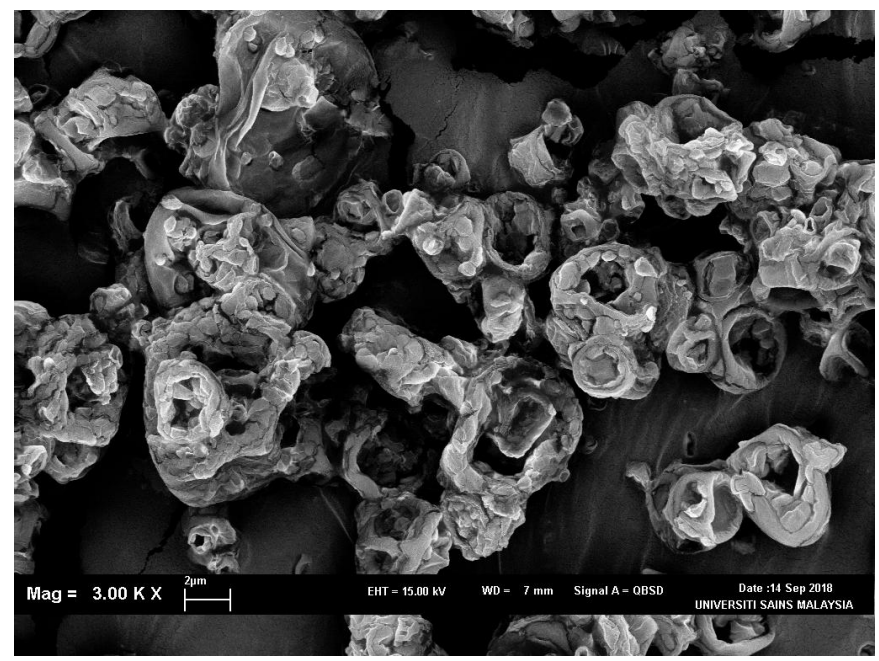

Figure 6: SEM image of chitosan NP produced.

\section{CONCLUSION}

Chitosan NP can be widely used for different applications, depending on their characteristics. Bigger particles can be used for topical drug delivery, whilst smaller NP are more suitable for systemic applications. In regards to this, the particle sizes of chitosan NP produced in this study are at bigger size range, therefore, they are more effective and useful for topical drug delivery compared to the intravenous drug delivery.

This paper has outlined the basic information needed in the production of chitosan $\mathrm{NP}$ as a guidance for researchers to choose the appropriate combination of components. The equation produced from the DoE software as described earlier can be used for this purpose. As shown in this study, acetic acid and chitosan flakes concentrations positively influenced the particles size, whilst the TPP concentration adversely affect the particles size. In general, higher concentration of acetic acid will dissolve chitosan flakes at a better rate and subsequently produce smaller particles. This is supported by the negative sign of the interaction between components A and B in Equation 1 (-1798.01AB), highlighting that the interaction between these two factors will produce small particles.

A more detailed study that investigates the drug loading ability of the NP, the drug release profile and the safety of the NP through in-vitro cell study is currently ongoing. The results are expected to provide further insights on the potentials of chitosan NP as a drug delivery vehicle. 


\section{ACKNOWLEDGEMENTS}

This study was funded by Universiti Sains Malaysia (USM) under the USM Short Term Grant (304.PFARMASI.6315097) entitled Development of Nanoparticles for Photodynamic Therapy Application 2018-2020.

\section{CONFLICT OF INTEREST}

The authors declare that there are no conflicts of interest.

\section{REFERENCES}

1. Benstead R. (2019). The future of the nanoparticle. Filt. Sep., 56(1), 20-21. https://doi.org/10.1016/S0015-1882(19)30035-7

2. Khadka, P. et al. (2014). Pharmaceutical particle technologies: An approach to improve drug solubility, dissolution and bioavailability. Asian J. Pharm. Sci., 9(6), 304-316. https://doi.org/10.1016/j.ajps.2014.05.005

3. Kemin, L. V. \& Chin, S-F. (2020). Amino-starch nanoparticles as controlled release nanocarriers for curcumin. J. Phys. Sci., 31(2), 1-14. https://doi.org/10.21315/ jps2020.31.2.1

4. Patra, J. K. et al. (2018). Nano based drug delivery systems: Recent developments and future prospects. J. Nanobiotech., 16(1), 71-80. https://doi.org/10.1186/ s12951-018-0392-8

5. Kakhaki, Z. M., Youzbashi, A. \& Naderi, N. (2015). Effect of milling energy and process ordering on the morphologies and optical properties of $\mathrm{ZnO}$ nanoparticles obtained through a mechanochemical technique. J. Phys. Sci., 26(2), 63-72.

6. Kahdestani, S. A., Shahriari, M. H. \& Abdouss, M. (2020). Synthesis and characterization of chitosan nanoparticles containing teicoplanin using sol-gel. Polym. Bull., https://doi.org/10.1007/s00289-020-03134-2

7. Aiping, Z. et al. (2006). Synthesis and characterization of N-succinyl-chitosan and its self-assembly of nanospheres. Carbohyd. Polym., 66(2), 274-279. https://doi.org/10.1016/j.carbpol.2006.03.014

8. Nagpal, K., Singh, S. K. \& Mishra, D. N. (2010). Chitosan nanoparticles: A promising system in novel drug delivery. Chem. Pharm. Bull., 58(11), 1423-1430. https://doi.org/10.1248/cpb.58.1423

9. Saeed, R. M., Dmour, I. \& Taha, M. O. (2020). Stable chitosan-based nanoparticles using polyphosphoric acid or hexametaphosphate for tandem ionotropic/covalent crosslinking and subsequent investigation as novel vehicles for drug delivery. Front. Bioeng. Biotech., 8(4), 1-10. https://doi.org/10.3389/fbioe.2020.00004

10. Abdelgawad, A. M.\&Hudson, S. M.(2019). Chitosannanoparticles: Polyphosphates cross-linking and protein delivery properties. Int. J. Biol. Macromol., 136, 133142. https://doi.org/10.1016/j.ijbiomac.2019.06.062 
11. Rinaudo, M. P. \& Desbrieres, J. (1999). Solubilization of chitosan in strong acid medium. Int. J. Polym. Anal. Ch., 5(3), 267-276. https://doi.org/10.1080/ 10236669908009742

12. Giri, T. K. (2016). Nanoarchitectured polysaccharide-based drug carrier for ocular therapeutics. In (Eds.) Holban, A. M. \& Grumezescu, A. M., Nanoarchitectonics for smart delivery and drug targeting. New York: William Andrew Publishing, 119-141.

13. Loutfy, S. A. et al.. (2016). Synthesis, characterization and cytotoxic evaluation of chitosan nanoparticles: In vitro liver cancel model. Adv. Nat. Sci-Nanosci., 7(3), 035008. https://doi.org/10.1088/2043-6262/7/3/035008

14. Lammel, T. et al. (2019). Endocytosis, intracellular fate, accumulation, and agglomeration of titanium dioxide $\left(\mathrm{TiO}_{2}\right)$ nanoparticles in the rainbow trout liver cell line RTL-W1. Environ. Sci. Pollut. R., 26(15), 15354. https://doi.org/ 10.1007/s11356-019-04856-1

15. Chu, Z. et al. (2014). Unambiguous observation of shape effects on cellular fate of nanoparticles. Sci. Rep. UK, 4(1), 4495. https://doi.org/10.1038/srep04495

16. Mohammed, M. A. et al. (2017). An overview of chitosan nanoparticles and its application in non-parenteral drug delivery. Pharm., 9(4), 53. https://doi.org/ 10.3390/pharmaceutics9040053 